\title{
Optimization of Biodynamic Seated Human Models Using Genetic Algorithms
}

\author{
Wael Abbas ${ }^{1 *}$, Ossama B. Abouelatta ${ }^{2}$, Magdi El-Azab ${ }^{3}$, Mamdouh Elsaidy ${ }^{4}$, Adel A. Megahed ${ }^{5}$ \\ ${ }^{1}$ Engineering Physics and Mathematics Department, Faculty of Engineering (Mataria), Helwan University, \\ Cairo, Egypt \\ ${ }^{2}$ Production Engineering and Mechanical Design Department, Faculty of Engineering, Mansoura University, \\ Mansoura, Egypt \\ ${ }^{3,4}$ Mathematics and Engineering Physics Department, Faculty of Engineering, Mansoura University, \\ Mansoura, Egypt \\ ${ }^{5}$ Mathematics and Engineering Physics Department, Faculty of Engineering, Cairo University, Cairo, Egypt \\ E-mail:wael_abass@hotmail.com \\ Received May 16, 2010; revised July 21, 2010; accepted August 4, 2010
}

\begin{abstract}
Many biodynamic models have been derived using trial and error curve-fitting technique, such that the error between the computed and measured biodynamic response functions is minimum. This study developed a biomechanical model of the human body in a sitting posture without backrest for evaluating the vibration transmissibility and dynamic response to vertical vibration direction. In describing the human body motion, a three biomechanical models are discussed (two models are 4-DOF and one model 7-DOF). Optimization software based on stochastic techniques search methods, Genetic Algorithms (GAs), is employed to determine the human model parameters imposing some limit constraints on the model parameters. In addition, an objective function is formulated comprising the sum of errors between the computed and actual values (experimental data). The studied functions are the driving-point mechanical impedance, apparent mass and seatto-head transmissibility functions. The optimization process increased the average goodness of fit and the results of studied functions became much closer to the target values (Experimental data). From the optimized model, the resonant frequencies of the driver parts computed on the basis of biodynamic response functions are found to be within close bounds to that expected for the human body.
\end{abstract}

Keywords: Biodynamic Response, Seated Human models, Simulation, Genetic algorithms

\section{Introduction}

Recently, many people have focused their attention on the ride quality of vehicle which is directly related to driver fatigue, discomfort, and safety. As traveling increases, the driver is more exposed to vibration mostly originating from the interaction between the road and vehicle. Whole-body vibration occurs in transportation and when near heavy machinery [1]. The vibrations cause the operator's whole body to vibrate, as opposed to just one part of their body, says their hand or foot. Harmful effects of whole-body vibration are experienced when the exposure time is longer than the recommended standard set by ISO 2631-1 [2].

Biodynamic responses of seated human occupant exposed to vibration have been widely characterized to define frequency-weightings for assessment of exposure, to identify human sensitivity and perception of vibration, and to develop seated body models [3]. The biodynamic response of the human body exposed to vibration have been invariably characterized through measurement of force motion relationship at the point of entry of vibration "To-the-body response function", and transmission of vibration to different body segments "Through-thebody response function". Considering that the human body is a complex biological system, the "To-the-body" response function is conveniently characterized through non-invasive measurements at the driving point alone. The vast majority of the reported studies on biodynamic response to whole-body vibration have considered vibration along the vertical axis alone.

The reported studies on biodynamic responses under 
vertical vibration are thoroughly reviewed, specifically their response characteristics, experimental conditions, and the measured data. The biodynamic response characteristics reported in terms of either the driving-point mechanical impedance (DPMI) or apparent mass (APMS), and the seat-to-head transmissibility (STHT) are classified under different experimental conditions used in the study.

In early studies, various biodynamic models have been developed to depict human motion from single-DOF to multi-DOF models. These models can be divided as distributed (finite element) models, lumped parameter models and multi-body models. The distributed model treats the spine as a layered structure of rigid elements, representing the vertebral bodies, and deformable elements representing the intervertebral discs by the finite element method. Multi-body human models are made of several rigid bodies interconnected by pin (two-dimensional) or ball and socket (three-dimensional) joints, and can be further separated into kinetic and kinematic models.

The lumped parameter models consider the human body as several rigid bodies and spring-dampers. This type of model is simple to analyze and easy to validate with experiments. However, the disadvantage is the limitation to one-directional analysis. Coermann [4], measured the driving-point impedance of the human body and suggested 1-DOF model. Suggs et al. [5] developed a 2-DOF human body. It was modeled as a damped spring-mass system to build a standardized vehicle seat testing procedure. A 3-DOF analytical model for a tractor seat suspension system is presented by Tewari et al. [6]. It was observed that the model could be employed as a tool in selection of optimal suspension parameters for any other type of vehicles. Boileau et al. [7] used an optimization procedure to establish a 4-DOF human model based on test data. In addition, Zong and Lam [8] validated a 4-DOF nonlinear model originating from Liu et al. [9].

Furthermore, Muksian and Nash [10] presented a 6DOF nonlinear model dedicated to the analysis of vibration imposed on a seated human. This model was modified by Patil et al. [11], who suggested a 7-DOF model. This model was further incorporated with a tractor model to evaluate vibration responses of an occupant-tractor system. A complete study on lumped-parameter models for seated human under vertical vibration excitation has been carried out by Liang and Chiang [12], based on analytical study and experimental validation.

On the other hand, GA optimization is used by Baumal et al. [13] to determine both active control and passive mechanical parameters of a vehicle suspension system, to minimize the extreme acceleration of the passen- ger's seat, subjected to constraints representing the required road holding ability and suspension working space. The GA is used to solve the problem and results were compared to those obtained by simulated annealing technique and found to yields similar performance measures.

It is clear that the lumped-parameter model is probably one of the most popular analytical methods in the study of biodynamic responses of seated human subjects, though it is limited to one-directional analysis. However, vertical vibration exposure of the driver is our main concern. Therefore, this paper carries out a thorough survey of literature on the lumped-parameter models for seated human subjects exposed to vertical vibration.

This work aims to develop a biomechanical model of the human body in a sitting posture without backrest for evaluating the vibration transmissibility and dynamic response to vertical vibration direction.

\section{Biodynamic Response of the Human Body}

The biodynamic response of a seated human body exposed to whole-body vibration can be broadly categorized into two types. The first category "To-the-body" force motion interrelation as a function of frequency at the human-seat interface, expressed as the driving-point mechanical impedance or the apparent mass. The second category "Through-the-body" response function, generally termed as seat-to-head transmissibility for the seated occupant.

The DPMI relates the driving force and resulting velocity response at the driving point (the seat-buttocks interface), and is given by [3]:

$$
Z(j \omega)=\frac{F(j \omega)}{V(j \omega)}=\frac{F(j \omega)}{X^{\bullet}(j \omega)}
$$

where, $Z(j \omega)$ is the complex DPMI, $F(j \omega)$ and $V(j \omega)$ or $X^{\bullet}(j \omega)$ are the driving force and response velocity at the driving point, respectively. $\omega$ is the angular frequency in $\mathrm{rad} / \mathrm{s}$, and $j=\sqrt{-1}$ is the complex phasor.

In a similar manner, the apparent mass response relates the driving force to the resulting acceleration response, and is given by [14]:

$$
A P M S(j \omega)=\frac{F(j \omega)}{a(j \omega)}
$$

where, $a(j \omega)$ is the acceleration response at the driving point. The magnitude of $A P M S$ offers a simple physical interpretation as it is equal to the static mass of the human body supported by the seat at very low frequencies, when the human body resembles that of a rigid 
mass. The above two functions are frequently used interchangeably, due to their direct relationship that given by:

$$
\operatorname{APMS}(j \omega)=\frac{D P M I(j \omega)}{j \omega}
$$

The biodynamic response characteristics of seated occupants exposed to whole body vibration can also be expressed in terms of seat-to-head transmissibility, which is termed as "through-the-body" response function. Unlike the force-motion relationship at the driving-point, the STHT function describes the transmission of vibration through the seated body. The STHT response function is expressed as:

$$
H(j \omega)=\frac{a_{H}(j \omega)}{a(j \omega)}
$$

where, $H(j \omega)$ is the complex STHT, $a_{H}(j \omega)$ is the response acceleration measured at the head of seated occupant, and $a(j \omega)$ is the acceleration response at the driving point. The above three functions have been widely used to characterize the biodynamic responses of the seated human subjects exposed to whole body vibration.

\section{Experimental Data}

Many mathematical models on the study of biodynamic responses of seated human subjects have been published based on individual test data over the years. However, significant variation is known to exist between various data sets. Such variation may be partly attributed to the differences associated with the methodology, experimental conditions or subject population used by different investigators.

\subsection{Basic Assumptions on Experimental Data}

The biodynamic of seated human subjects exposed to vertical vibration has been widely assessed in terms of STHT, DPMI, and APMS. The first function refers to the transmission of motion through the body, while the other two relate the force and motion at the point of vibration input to the body. A variety of test data used to characterize these response functions has been established using widely varied test conditions. This has resulted in considerable discrepancies among the data. To avoid these discrepancies, a preliminary conclusion was reached that any attempt to define generalized values might not be appropriate unless it could be defined specifically for a particular application or within a limited and welldefined range of situations [12].
Data sets satisfying the following requirements are selected for the synthesis of biodynamic characteristics of the seated human subjects [15-17].

- A human subject is considered to be sitting erect without backrest support, irrespective of the hands' position.

- $\quad$ Body masses will be limited within 49-94 kg.

- $\quad$ Feet are supported and vibrated.

- Analysis is constrained to the vertical direction.

- Vibration excitation amplitudes are below $5 \mathrm{~m} / \mathrm{s}^{2}$, with the nature of excitation specified as being sinusoidal wave.

- Excitation frequency range is limited to $0.5-20 \mathrm{~Hz}$.

\subsection{Experimental Results}

While vertical DPMI, APAS, and STHT characteristics were not measured as part of this study, applicable target values were defined on the basis of a synthesis of published data Boileau [14], Liang et al. [12,17] and $\mathrm{Wu}$ [18]. Figure 1 shows upper, lower, and target values of DPMI, APMS, and STHT magnitude established as target values within $0.5-20 \mathrm{~Hz}$ frequency range, respectively.

\section{Biomechanical Modeling}

The human body in a sitting posture can be modeled as a mechanical system that is composed of several rigid bodies interconnected by springs and dampers. In this study, three types of biomechanical models are discussed to describe the vertical response: 4-DOF Wan and Schimmels model, 4-DOF Boileau and Rakheja model, and 7-DOF Patil and Palanichamy model as shown in Figure 2.

\subsection{Wan and Schimmels 4-DOF Model}

In this model, the seated human body was constructed with four separate mass segments interconnected by five sets of springs and dampers, with a total human mass of $60.67 \mathrm{~kg}$ [19]. The four masses represent the following body segments: head and neck $\left(m_{1}\right)$, upper torso $\left(m_{2}\right)$, lower torso $\left(m_{3}\right)$, and thighs and pelvis $\left(m_{4}\right)$. The arms and legs are combined with the upper torso and thigh, respectively. The stiffness and damping properties of thighs and pelvis are $\left(k_{5}\right)$ and $\left(c_{5}\right)$, the lower torso are $\left(k_{4}\right)$ and $\left(c_{4}\right)$, upper torso are $\left(k_{2}, k_{3}\right)$ and $\left(c_{2}, c_{3}\right)$, and head are $\left(k_{1}\right)$ and $\left(c_{1}\right)$. The schematic of the model is shown in Figure 2(a), and biomechanical parameters of the model are listed in Table $\mathbf{1 .}$

The equations of motion of the human-body can be obtained as follows: 


$$
\left\{\begin{array}{l}
m_{1} x_{1}^{\bullet \bullet}=-c_{1}\left(x_{1}^{\bullet}-x_{2}^{\bullet}\right)-k_{1}\left(x_{1}-x_{2}\right) \\
m_{2} x_{2}^{\bullet \bullet}=c_{1}\left(x_{1}^{\bullet}-x_{2}^{\bullet}\right)+k_{1}\left(x_{1}-x_{2}\right)-c_{2}\left(x_{2}^{\bullet}-x_{3}^{\bullet}\right) \\
-k_{2}\left(x_{2}-x_{3}\right)-c_{3}\left(x_{2}^{\bullet}-x_{4}^{\bullet}\right)-k_{3}\left(x_{2}-x_{4}\right) \\
m_{3} x_{3}^{\bullet \bullet}=c_{2}\left(x_{2}^{\bullet}-x_{3}^{\bullet}\right)+k_{2}\left(x_{2}-x_{3}\right) \\
-c_{4}\left(x_{3}^{\bullet}-x_{4}^{\bullet}\right)-k_{4}\left(x_{3}-x_{4}\right) \\
m_{4} x_{4}^{\bullet \bullet}=c_{4}\left(x_{3}^{\bullet}-x_{4}^{\bullet}\right)+k_{4}\left(x_{3}-x_{4}\right)+c_{3}\left(x_{2}^{\bullet}-x_{4}^{\bullet}\right) \\
+k_{3}\left(x_{2}-x_{4}\right)-c_{5}\left(x_{4}^{\bullet}-x_{s e}^{\bullet}\right)-k_{5}\left(x_{4}-x_{s e}\right)
\end{array}\right.
$$

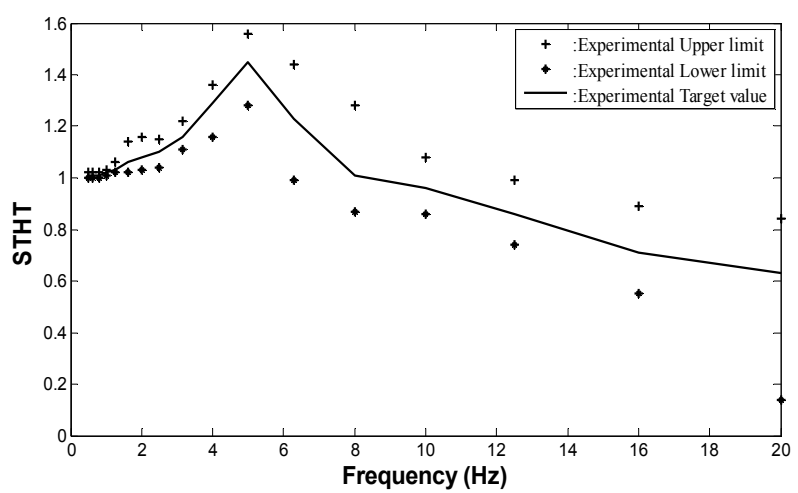

(a)

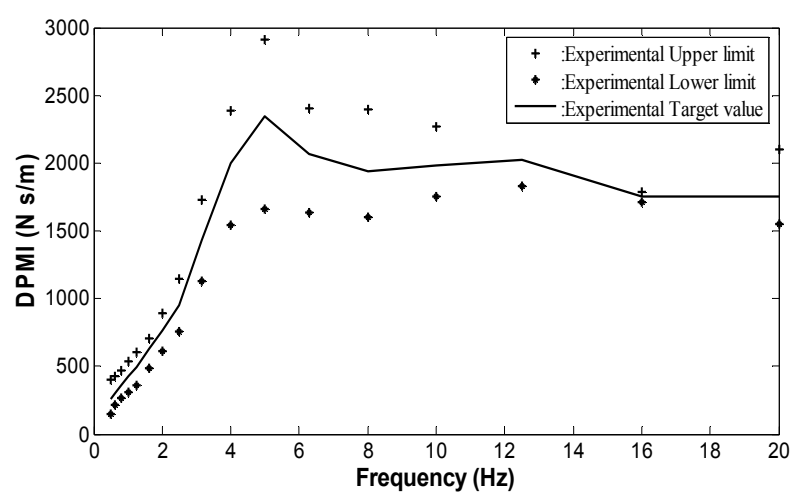

(b)

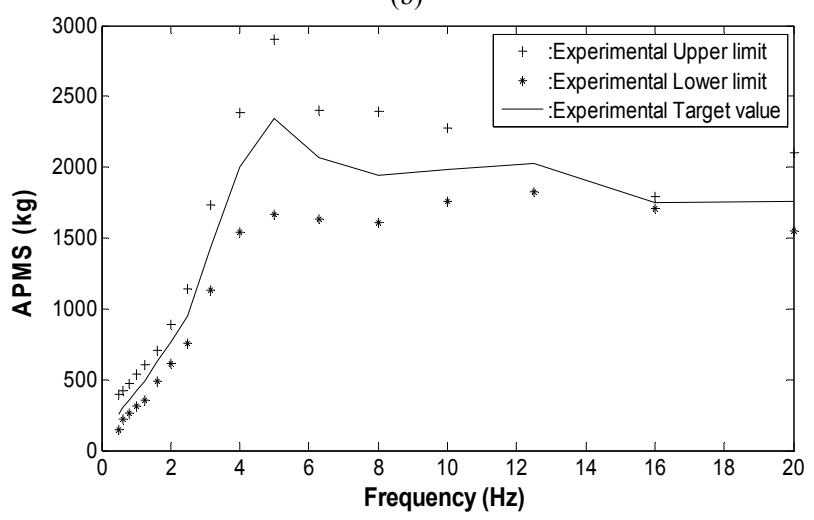

(c)

Figure 1. Experimental data of (a) STHT; (b) DPMI; and (c) APMS.

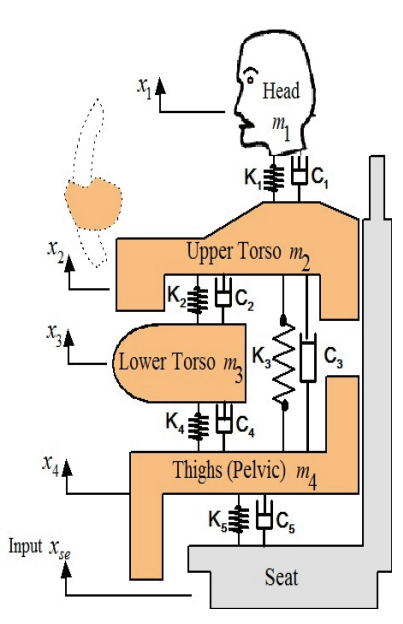

(a)

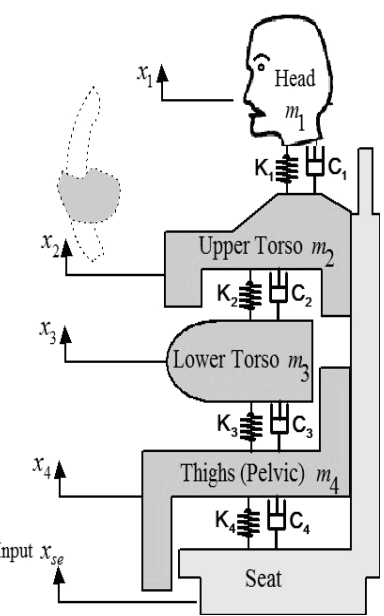

(b)

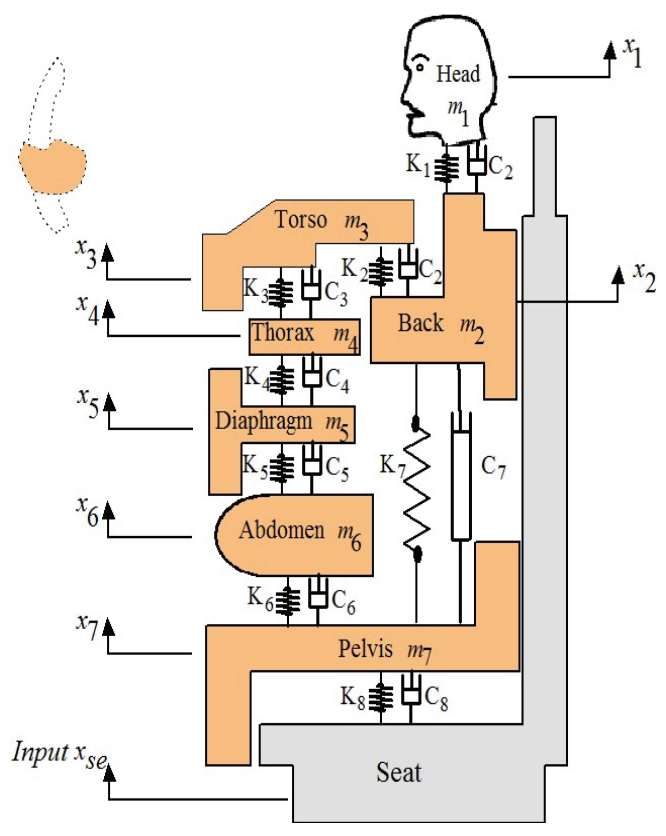

(c)

Figure 2. Biomechanical models. (a) Wan and Schimmels 4-DOF model; (b) Boileau and Rakheja 4-DOF model; and (c) Patil and Palanichamy 7-DOF model.

\subsection{Boileau and Rakheja 4-DOF Model}

The human-body consists of four mass segments interconnected by four sets of springs and dampers with a total mass of $55.2 \mathrm{~kg}$ [7], as shown in Figure 2(b). The four masses represent the following four body segments: the head and neck $\left(m_{1}\right)$, the chest and upper torso $\left(m_{2}\right)$, the lower torso $\left(m_{3}\right)$, and the thighs and pelvis in contact with the seat $\left(m_{4}\right)$. The mass due to lower legs and the feet is not included in this representation, assuming their negligible contributions to the biodynamic response of the seated body. The stiffness and damping properties of 
thighs and pelvis are $\left(k_{4}\right)$ and $\left(c_{4}\right)$, the lower torso are $\left(k_{3}\right)$ and $\left(c_{3}\right)$, upper torsos are $\left(k_{2}\right)$ and $\left(c_{2}\right)$, and head are $\left(k_{1}\right)$ and $\left(c_{1}\right)$. The biomechanical parameters of the model are listed in Table 2. The equation of motion of the human body can be obtained as follows:

$$
\left\{\begin{array}{l}
m_{1} x_{1}^{\bullet \bullet}=-c_{1}\left(x_{1}^{\bullet}-x_{2}^{\bullet}\right)-k_{1}\left(x_{1}-x_{2}\right) \\
m_{2} x_{2}^{\bullet \bullet}=c_{1}\left(x_{1}^{\bullet}-x_{2}^{\bullet}\right)+k_{1}\left(x_{1}-x_{2}\right)-c_{2}\left(x_{2}^{\bullet}-x_{3}^{\bullet}\right) \\
-k_{2}\left(x_{2}-x_{3}\right) \\
m_{3} x_{3}^{\bullet \bullet}=c_{2}\left(x_{2}^{\bullet}-x_{3}^{\bullet}\right)+k_{2}\left(x_{2}-x_{3}\right)-c_{3}\left(x_{3}^{\bullet}-x_{4}^{\bullet}\right) \\
-k_{3}\left(x_{3}-x_{4}\right) \\
m_{4} x_{4}^{\bullet \bullet}=c_{3}\left(x_{2}^{\bullet}-x_{4}^{\bullet}\right)+k_{3}\left(x_{2}-x_{4}\right)-c_{4}\left(x_{4}^{\bullet}-x_{s e}^{\bullet}\right) \\
-k_{4}\left(x_{4}-x_{s e}\right)
\end{array}\right.
$$

\subsection{Patil and Palanichamy 7-DOF Model}

Based on Muksian's 6-DOF model, a 7-DOF nonlinear model was developed by Patil and Palanichamy [11]. In this model, the human body consists of seven mass segments interconnected by eight sets of springs and dampers, with total mass of $80 \mathrm{~kg}$. The seven masses represent the following body segments: head and neck $\left(m_{1}\right)$, back $\left(m_{2}\right)$, upper torso $\left(m_{3}\right)$, thorax $\left(m_{4}\right)$, diaphragm $\left(m_{5}\right)$, abdomen $\left(m_{6}\right)$ and thighs and pelvis $\left(m_{7}\right)$. The arms and legs are combined with the upper torso and thigh, respectively. The stiffness and damping properties of thighs and pelvis are $\left(\mathrm{k}_{8}\right)$ and $\left(\mathrm{c}_{8}\right)$, abdomen are $\left(\mathrm{k}_{6}\right)$ and $\left(\mathrm{c}_{6}\right)$, the diaphragm are $\left(\mathrm{k}_{5}\right)$ and $\left(\mathrm{c}_{5}\right)$, the thorax are $\left(\mathrm{k}_{4}\right)$ and $\left(\mathrm{c}_{4}\right)$, the torso are $\left(\mathrm{k}_{2}, \mathrm{k}_{3}\right)$ and $\left(\mathrm{c}_{2}, \mathrm{c}_{3}\right)$, back are $\left(\mathrm{k}_{7}\right)$ and $\left(c_{7}\right)$, and head are $\left(k_{1}\right)$ and $\left(c_{1}\right)$. The schematic of the model is shown in Figure 2(c), and biomechanical parameters of the model are listed in Table 3.

The equation of motion of the human-body can be obtained as follows:

$$
\left\{\begin{array}{l}
m_{1} x_{1}^{\bullet \bullet}=-c_{1}\left(x_{1}^{\bullet}-x_{2}^{\bullet}\right)-k_{1}\left(x_{1}-x_{2}\right) \\
m_{2} x_{2}^{\bullet \bullet}=c_{1}\left(x_{1}^{\bullet}-x_{2}^{\bullet}\right)+k_{1}\left(x_{1}-x_{2}\right)-c_{7}\left(x_{2}^{\bullet}-x_{7}^{\bullet}\right) \\
-k_{7}\left(x_{2}-x_{7}\right)-c_{2}\left(x_{2}^{\bullet}-x_{3}^{\bullet}\right)-k_{2}\left(x_{2}-x_{3}\right) \\
m_{3} x_{3}^{\bullet \bullet}=c_{2}\left(x_{2}^{\bullet}-x_{3}^{\bullet}\right)+k_{2}\left(x_{2}-x_{3}\right)-c_{3}\left(x_{3}^{\bullet}-x_{4}^{\bullet}\right) \\
-k_{3}\left(x_{3}-x_{4}\right) \\
m_{4} x_{4}^{\bullet \bullet}=c_{3}\left(x_{3}^{\bullet}-x_{4}^{\bullet}\right)+k_{3}\left(x_{3}-x_{4}\right)-c_{4}\left(x_{4}^{\bullet}-x_{5}^{\bullet}\right) \\
-k_{4}\left(x_{4}-x_{5}\right) \\
m_{5} x_{5}^{\bullet \bullet}=c_{4}\left(x_{4}^{\bullet}-x_{5}^{\bullet}\right)+k_{4}\left(x_{4}-x_{5}\right)-c_{5}\left(x_{5}^{\bullet}-x_{6}^{\bullet}\right) \\
-k_{5}\left(x_{5}-x_{6}\right) \\
m_{6} x_{6}^{\bullet \bullet}=c_{5}\left(x_{5}^{\bullet}-x_{6}^{\bullet}\right)+k_{5}\left(x_{5}-x_{6}\right)-c_{6}\left(x_{6}^{\bullet}-x_{7}^{\bullet}\right) \\
-k_{6}\left(x_{6}-x_{7}\right) \\
m_{7} x_{7}^{\bullet \bullet}=c_{6}\left(x_{6}^{\bullet}-x_{7}^{\bullet}\right)+k_{6}\left(x_{6}-x_{7}\right)+c_{7}\left(x_{2}^{\bullet}-x_{7}^{\bullet}\right) \\
+k_{7}\left(x_{2}-x_{7}\right)-c_{8}\left(x_{7}^{\bullet}-x_{s e}^{\bullet}\right)-k_{8}\left(x_{7}-x_{s e}\right)
\end{array}\right.
$$

\section{Estimation of Biodynamic Response Characteristics}

There are two methods to solve system equations of motion; time domain and frequency domain. Frequency domain solutions are often of more interest than the time history and can usually be performed more conveniently than in the time domain. However, for the solutions to be applicable, the equations must either be linear, or linearized. Frequency domain analysis employs the Fourier transformation.

The system equations of motion, Equations (5-7), for the model can be expressed in matrix form as follows:

$$
[M]\left\{x^{\bullet \bullet}\right\}+[C]\left\{x^{\bullet}\right\}+[K]\{x\}=\{f\}
$$

where, $[M],[C]$ and $[K]$ are $n \times n$ mass, damping, and stiffness matrices, respectively; $\{f\}$ is the force vector due to external excitation.

By taking the Fourier transformation of equation (8), the following matrix form of equation can be obtained:

$$
\{X(j \omega)\}=\left[[K]-\omega^{2}[M]+j \omega[C]\right]^{-1}\{F(j \omega)\}
$$

where, $\{X(j \omega)\}$ and $\{F(j \omega)\}$ are the complex Fourier transformation vectors of $\{x\}$ and $\{f\}$ respectively. $\omega$ is the excitation frequency. Vector $\{X(j \omega)\}$ contains complex displacement responses of $\mathrm{n}$ mass segments as a function of $\omega$

$$
\left(\left\{x_{1}(j \omega), x_{2}(j \omega), x_{3}(j \omega), \ldots \ldots x_{n}(j \omega)\right\}\right) .
$$

$\{F(j \omega)\}$, consists of complex excitation forces on the mass segments as a function of $\omega$ as well.

The driving-point mechanical impedance is defined as the ratio of driving force (summation of spring and damping forces between pelvis and seat) to the drivingpoint velocity (input velocity of the seat). Accordingly, DPMI can be represented as follows (e.g. Boileau and Rakheja, model):

$$
\begin{aligned}
& \operatorname{DPMI}(j \omega)= \\
& \left|\left(c_{4}+\frac{k_{4}}{j \omega}\right) \frac{x_{4}(j \omega)}{x_{0}(\omega)}-\left(c_{4}+\frac{k_{4}}{j \omega}\right)\right|
\end{aligned}
$$

Seat-to-head transmissibility is defined as the ratio of output responses (head) to input excitation.

$$
\operatorname{STHT}(j \omega)=\frac{x_{1}(j \omega)}{x_{0}(j \omega)}
$$

Apparent mass, can be expressed in terms of DPMI, Equation (3), as follows (e.g. Boileau and Rakheja model):

$$
\begin{aligned}
& \operatorname{APMS}(j \omega)=\left|\frac{D P M I(j \omega)}{j \omega}\right| \\
& =\left|\left(\frac{c_{4}}{j \omega}+\frac{k_{4}}{-\omega^{2}}\right) \frac{x_{4}(j \omega)}{x_{0}(j \omega)}-\left(\frac{c_{4}}{j \omega}+\frac{k_{4}}{-\omega^{2}}\right)\right|
\end{aligned}
$$


Table 1. The biomechanical parameters of the Wan and Schimmels model (Before and after optimization).

\begin{tabular}{|c|c|c|c|c|}
\hline \multirow{2}{*}{$\begin{array}{c}\text { Mass } \\
(\mathrm{kg})\end{array}$} & \multicolumn{2}{|c|}{$\begin{array}{l}\text { Damping coefficient } \\
\text { (N.s/m) }\end{array}$} & \multicolumn{2}{|c|}{ Spring constant $(\mathrm{N} / \mathrm{m})$} \\
\hline & Before & After & Before & After \\
\hline $\begin{array}{l}m_{1}= \\
4.17\end{array}$ & $c_{1}=250$ & $c_{1}=310$ & $\begin{array}{l}k_{1}= \\
134400\end{array}$ & $\begin{array}{l}k_{1}= \\
166990\end{array}$ \\
\hline$m_{2}=15$ & $c_{2}=200$ & $c_{2}=200$ & $\begin{array}{l}k_{2}= \\
10000\end{array}$ & $\begin{array}{l}k_{2}= \\
10000\end{array}$ \\
\hline $\begin{array}{l}m_{3}= \\
5.5\end{array}$ & $c_{3}=909.1$ & $\begin{array}{l}c_{3}= \\
909.1\end{array}$ & $\begin{array}{l}k_{3}= \\
192000\end{array}$ & $\begin{array}{l}k_{3}= \\
144000\end{array}$ \\
\hline$m_{4}=36$ & $c_{4}=330$ & $c_{4}=330$ & $\begin{array}{l}k_{4}= \\
20000\end{array}$ & $\begin{array}{l}k_{4}= \\
20000\end{array}$ \\
\hline- & $c_{5}=2475$ & $c_{5}=2475$ & $k_{5}=49340$ & $k_{5}=49340$ \\
\hline
\end{tabular}

\section{Development of Models}

Many biodynamic models have been derived using trial and error curve-fitting technique, such that the error between the computed and measured biodynamic response functions is minimum. Such curve-fitting methods may lead to a proper fit over a specific frequency range, but rarely provide good results when extended over a broad frequency range. Alternatively, nonlinear programming based optimization techniques may be effectively employed to determine the model parameters, involving the use of a constrained optimization algorithm in conjunction with well defined biodynamic response function [14]. A constrained objective function may be defined to minimize the error between the computed and the target values of specific biodynamic response function over a specific frequency range.

Optimization software based on stochastic techniques search methods, Genetic algorithms (GAs), is employed to determine the human model parameters imposing some limit constraints on the model parameters. An objective function is formulated comprising the sum of errors between the computed and of the driving-point mechanical impedance, apparent mass and seat-to-head transmissibility functions. The model thus derived can provide reasonable correlation with the impedance, apparent mass and transmissibility characteristics.

Starting with an assumed set of model parameters, the differential equations of motion are solved for unit displacement excitation to drive the driving-point mechanical impedance using Equation (1), apparent mass using Equation (3), and seat-to-head transmissibility using Equation (4). At each iteration of search, the sum of square errors defined by an objective function over the entire frequency range is examined, and the procedure is re-initiated with modified parameter values when the error exceeds that from the previous search. The search is terminated when the computed error approaches the minimum value.

\subsection{Objective Function}

The objective function is selected to comprise the squared sum errors associated with driving-point mechanical impedance $\left(U_{1}\right)$ apparent mass $\left(U_{2}\right)$ and seat-to-head transmissibility functions $\left(U_{3}\right)$ to minimize the error between the computed and the target values. This study used the classical weighted sum approaches to solve a multi-objective optimization problem as follows:

$$
O B J=W_{1} \cdot\left(U_{1}\right)+W_{2} \cdot\left(U_{2}\right)+W_{3} \cdot\left(U_{3}\right)
$$

where,

$$
\begin{gathered}
U_{1}=\sum_{i=1}^{n}\left[\left|\operatorname{DPMI}\left(j \omega_{i}\right)\right|-\left|\operatorname{DPMI}_{t}\left(j \omega_{i}\right)\right|\right]^{2}, \\
U_{2}=\sum_{i=1}^{n}\left[\left|A P M S\left(j \omega_{i}\right)\right|-\left|A P M S_{t}\left(j \omega_{i}\right)\right|\right]^{2}, \\
U_{3}=\sum_{i=1}^{n}\left[\left|\operatorname{STHT}\left(j \omega_{i}\right)\right|-\left|\operatorname{STHT}_{t}\left(j \omega_{i}\right)\right|\right]^{2}
\end{gathered}
$$

In the above equations $\left|D P M I_{t}\left(j \omega_{i}\right)\right|,\left|A P M S_{t}\left(j \omega_{i}\right)\right|$ and $\left|S T H T_{t}\left(j \omega_{i}\right)\right|$ are the target values of driving-point mechanical impedance, apparent mass, and seat-to-head transmissibility, respectively. The target values of DPMI, APMS, and STHT are illustrated in Figure 1. $W_{1}, W_{2}$ and $W_{3}$ are weighting factors to emphasize the relative importance of the terms.

The limit constraints are calculated as $\pm 25 \%$ variations about the biomechanical parameters of the three models.

\subsection{Evaluation of Biodynamic Seated Human Models}

To evaluate the prediction accuracy of each human model in comparison with experimental results from literature, the ratio of the root-mean-square error to the mean value is calculated with the following equation:

Goodness of fit $(\varepsilon)=\frac{\sqrt{\sum\left(\tau_{e}-\tau_{c}\right)^{2} /(N-2)}}{\sum \tau_{e} / N}$

where $\tau_{e}$ is the test datum, $\tau_{c}$ is the calculated result from each model, and $\mathrm{N}$ is the number of test data points used in the comparison. The fit of predicted results to test data is perfect when $\varepsilon$ is equal to 1 . The predictions on seat-to-head transmissibility, the driving-point mechanical impedance, and apparent mass for each lumped-parameter model will be compared using Equation (14) to obtain prediction accuracy.

\section{Results and Discussion}

\section{1) Wan and Schimmels modified model}

The solution of the constrained optimization problem, 
Equation (5), resulted in an optimized model parameters are listed in Table 1. Figure 3 presents a comparison of the driving-point mechanical impedance, apparent mass, and seat-to-head transmissibility with the target data, respectively. It is obvious that the developed model better fits target values compared with Wan and Schimmels model. The calculated goodness of fit for seat-to-head transmissibility is $92 \%$ compared to $90.9 \%$ for Wan and Schimmels model.

On the other hand, the developed model matches the target values better with a goodness of fit of $82.1 \%$ for

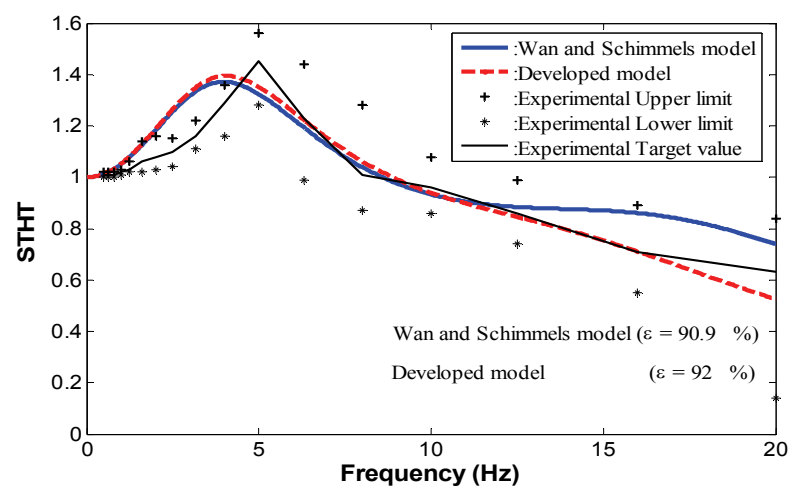

(a)

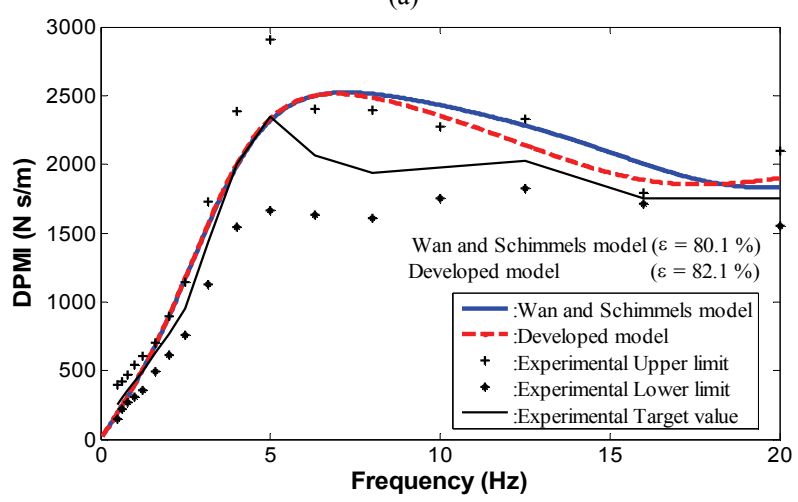

(b)

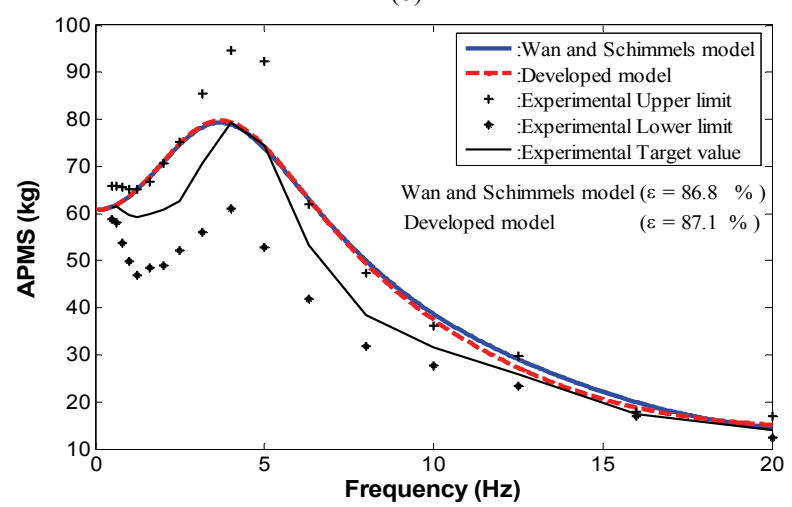

(c)

Figure 3. Comparison of biodynamic response characteristics for Wan model, and optimized model with the target data. driving-point mechanical impedance and $87.1 \%$ apparent mass compared to $80.1 \%$ and $86.8 \%$, for Wan and Schimmels model, respectively.

In addition, the peak values of the Wan and Schimmels model occur at $4 \mathrm{~Hz}$ for seat-to-head transmissibility, $3.7 \mathrm{~Hz}$ for apparent mass, and $7.2 \mathrm{~Hz}$ for drivingpoint mechanical impedance, whereas for the optimized model, they occur at 4.05, 3.8 and $6.9 \mathrm{~Hz}$, respectively.

\section{2) Boileau and Rakheja modified model}

The solution of the constrained optimization problem, Equation (6), resulted in optimized model parameters which are listed in Table 2.

Simulation results are illustrated in Figure 4. This figure presents a comparison of the driving-point mechanical impedance, apparent mass and seat-to-head transmissibility with the target data, respectively. It is obvious that the optimized model better fits target values than the Boileau and Rakheja model; with a goodness of fit for seat-to-head transmissibility is $80.6 \%$, compared to $76.8 \%$ for the Boileau and Rakheja model.

In addition, the developed model matches the target values better with a goodness of fit of $84 \%$ for driving-point mechanical impedance compared to $80.1 \%$ for Boileau and Rakheja model. Optimized model matches the target values better with a goodness of fit of $87 \%$ for apparent mass compared to $86.7 \%$ for the Boileau and Rakheja model.

On the other hand, the peak values of the Boileau and Rakheja model occur at $4.7 \mathrm{~Hz}$ for seat-to-head transmissibility, $4.6 \mathrm{~Hz}$ for apparent mass, and $5.5 \mathrm{~Hz}$ for driving-point mechanical impedance, whereas for the optimized model, they occur at 4.95, 4.55 and $5.9 \mathrm{~Hz}$, respectively.

\section{3) Patil and Palanichamy modified model}

In a similar way, the solution of the constrained optimization problem, Equation (7), resulted in an optimized of the following model parameters are listed in Table 3. Figure 5 presents a comparison of the driving-point mechanical impedance, apparent mass, and seat-to-head transmissibility with the target data, respectively. It was observed that the developed model better fits target values compared the Patil and Palanichamy model, with a goodness of fit for seat-to-head transmissibility is 35\% compared to $22 \%$ for Patil and Palanichamy model. On the other hand, the developed model matches the target values better with a goodness of fit of $13 \%$ for drivingpoint mechanical impedance compared to $1 \%$ for Patil and Palanichamy model. The developed model matches the target values with a goodness of fit of $34 \%$ for apparent mass compared to $0 \%$ for Patil and Palanichamy model. 
Table 2. The biomechanical parameters of the Boileau and Rakheja model (Before and after optimization).

\begin{tabular}{|c|c|c|c|c|}
\hline \multirow{2}{*}{ Mass (kg) } & \multicolumn{2}{|c|}{$\begin{array}{c}\text { Damping coefficient } \\
\text { (N.s/m) }\end{array}$} & \multicolumn{2}{|c|}{ Spring constant $(\mathrm{N} / \mathrm{m})$} \\
\hline & Before & After & Before & After \\
\hline $\begin{array}{l}m_{1}= \\
5.31\end{array}$ & $c_{1}=400$ & $c_{1}=460$ & $\begin{array}{l}k_{1}= \\
310000\end{array}$ & $\begin{array}{l}k_{1}= \\
356370\end{array}$ \\
\hline $\begin{array}{l}m_{2}= \\
28.49\end{array}$ & $c_{2}=4750$ & $c_{2}=5400$ & $\begin{array}{l}k_{2}= \\
183000\end{array}$ & $\begin{array}{l}k_{2}= \\
208570\end{array}$ \\
\hline $\begin{array}{l}m_{3}= \\
8.62\end{array}$ & $c_{3}=4585$ & $c_{3}=5190$ & $\begin{array}{l}k_{3}= \\
162800\end{array}$ & $\begin{array}{l}k_{3}= \\
187110\end{array}$ \\
\hline $\begin{array}{l}m_{4}= \\
12.78\end{array}$ & $c_{4}=2064$ & $c_{4}=2370$ & $k_{4}=90000$ & $\begin{array}{l}k_{4}= \\
103480\end{array}$ \\
\hline
\end{tabular}

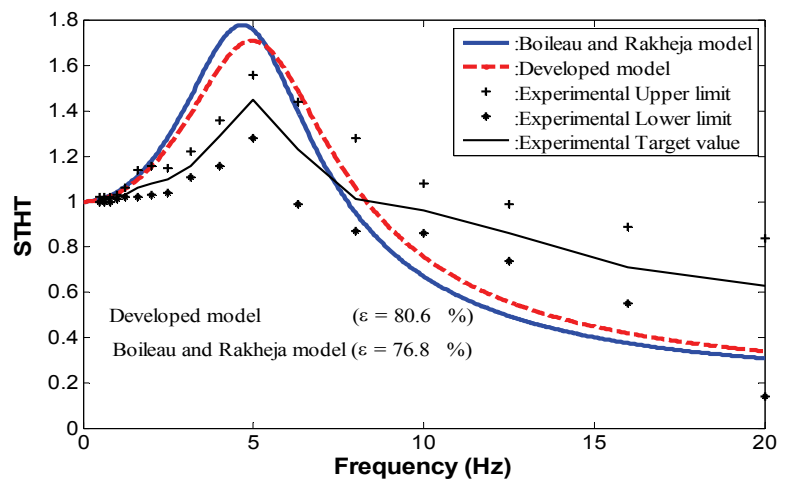

(a)

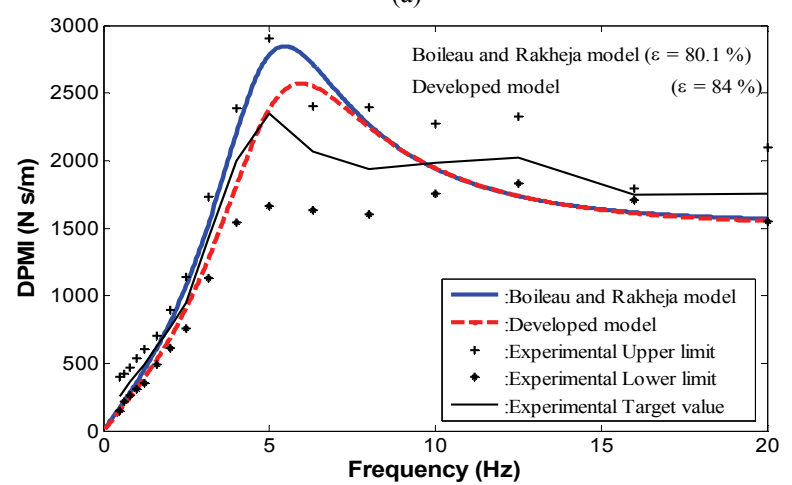

(b)

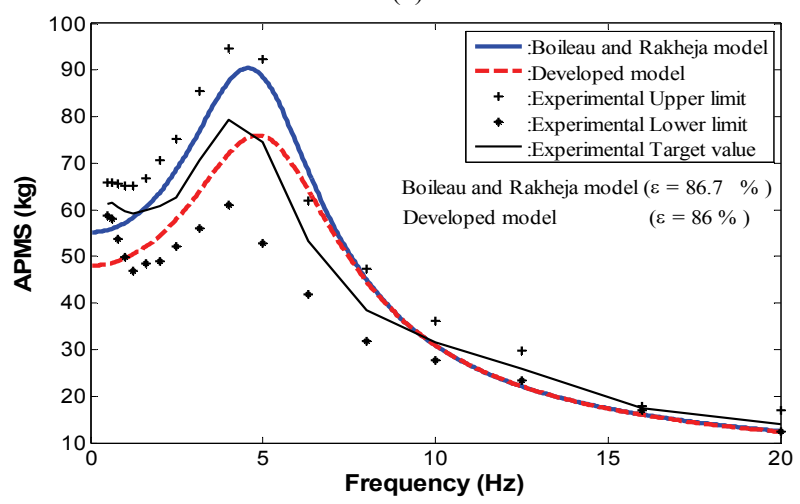

(c)

Figure 4. Comparison of biodynamic response characteristics for Boileau model, and optimized model with the target data.

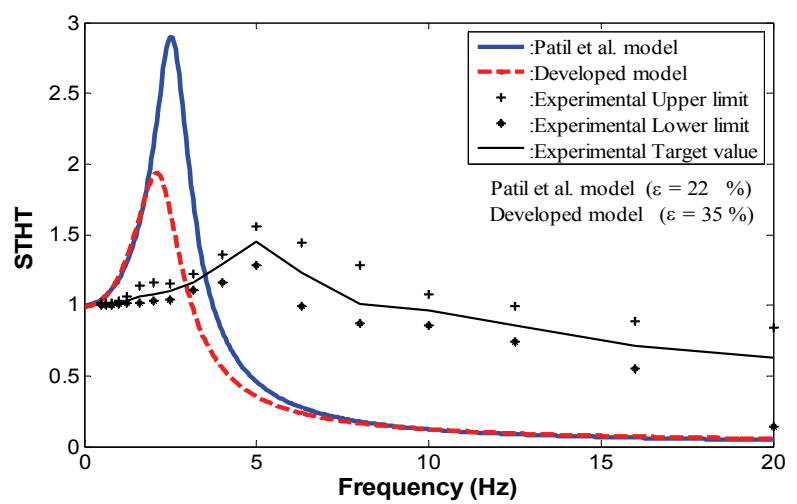

(a)

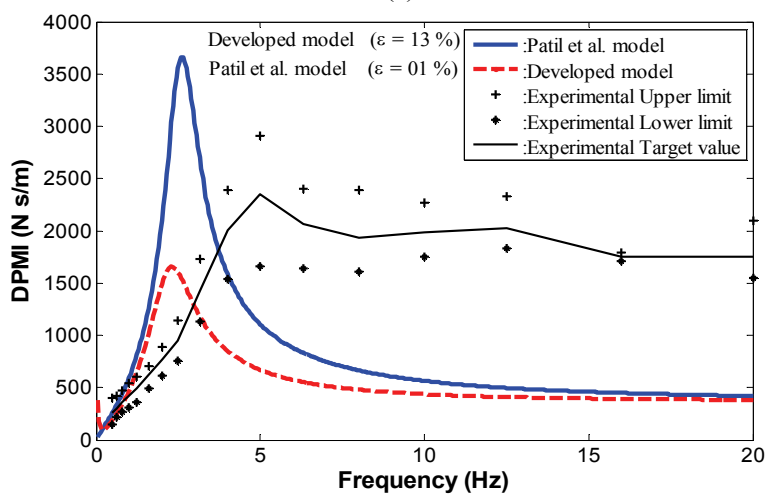

(b)

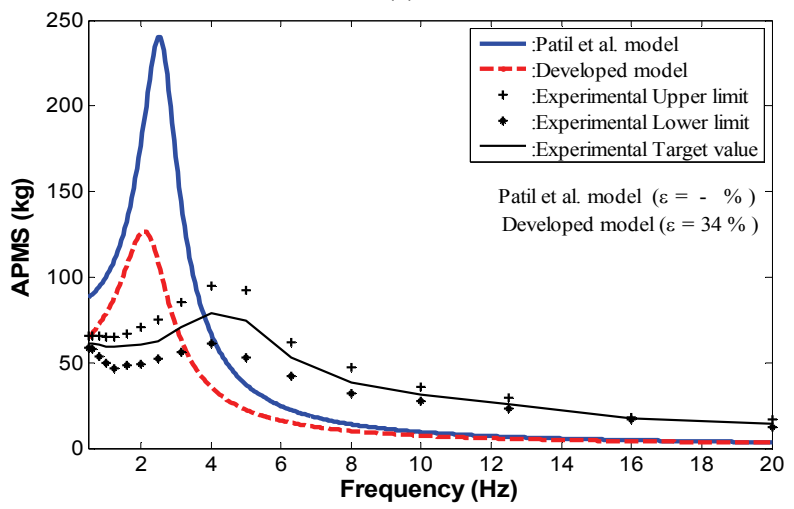

(c)

Figure 5. Comparison of biodynamic response characteristics for Patil model, and optimized model with the target data.

In addition, the peak values of Patil and Palanichamy model occur at $2.5 \mathrm{~Hz}$ for seat-to-head transmissibility, $2.5 \mathrm{~Hz}$ for apparent mass, and $2.6 \mathrm{~Hz}$ for driving-point mechanical impedance, whereas for the developed model, they occur at $2.1,2.1$ and $2.3 \mathrm{~Hz}$, respectively.

The simulations of the three lumped-parameter models listed in this study for seated human subjects exposed to vertical vibration exposure are listed in Table 4 . It is observed that the 4-DOF optimization of Wan and Schimmels can give the best estimation on seat-to-head transmissibility with goodness of fit values of $92 \%$. The 
Table 3. The biomechanical parameters of the Patil and Palanichamy model (Before and after optimization).

\begin{tabular}{|c|c|c|c|c|}
\hline \multirow{2}{*}{ Mass (kg) } & \multicolumn{2}{|c|}{$\begin{array}{c}\text { Damping coefficient } \\
\text { (N.s } / \mathrm{m})\end{array}$} & \multicolumn{2}{|c|}{ Spring constant $(\mathrm{N} / \mathrm{m})$} \\
\hline & Before & After & Before & After \\
\hline$m_{1}=5.55$ & $c_{1}=3651$ & $c_{1}=3542$ & $\begin{array}{l}k_{1}= \\
53640\end{array}$ & $\begin{array}{l}k_{1}= \\
41978\end{array}$ \\
\hline$m_{2}=6.94$ & $c_{2}=3651$ & $c_{2}=2685$ & $\begin{array}{l}k_{2}= \\
53640\end{array}$ & $\begin{array}{l}k_{2}= \\
40943\end{array}$ \\
\hline $\begin{array}{l}m_{3}= \\
33.33\end{array}$ & $c_{3}=298$ & $c_{3}=351$ & $k_{3}=8941$ & $k_{3}=1001$ \\
\hline $\begin{array}{l}m_{4}= \\
1.389\end{array}$ & $c_{4}=298$ & $c_{4}=237$ & $k_{4}=8941$ & $k_{4}=845$ \\
\hline $\begin{array}{l}m_{5}= \\
0.4629\end{array}$ & $c_{5}=298$ & $c_{5}=354$ & $k_{5}=8941$ & $k_{5}=1052$ \\
\hline$m_{6}=6.02$ & $c_{6}=298$ & $c_{6}=225$ & $k_{6}=8941$ & $k_{6}=1035$ \\
\hline \multirow[t]{2}{*}{$m_{7}=27.7$} & $c_{7}=3651$ & $c_{7}=2929$ & $\begin{array}{l}k_{7}= \\
53640\end{array}$ & $\begin{array}{l}k_{7}= \\
39575\end{array}$ \\
\hline & $c_{8}=378$ & $c_{8}=463$ & $\begin{array}{l}k_{8}= \\
25500\end{array}$ & $\begin{array}{l}k_{8}= \\
19325\end{array}$ \\
\hline
\end{tabular}

goodness of fit is $82.1 \%$ for driving-point mechanical impedance. On the other hand, the development model matches the target values better with a goodness of fit of $87.1 \%$ for apparent mass compared to all models.

\section{Conclusions and Recommendations}

A study on the biodynamic models of seated human subjects exposed to vertical vibration is carried out. A three lumped-parameter models from literature have also been analyzed and optimized using genetic algorithms to match an experimental data in terms of STH transmissibility, DPM impedance, and AP mass. It is shown that the optimized 4-DOF Wan and Schimmels model can give the best estimation on STH transmissibility, DPM impedance, AP mass with goodness of fit values of $91.2 \%, 82.1 \%$, and $87.1 \%$, respectively. In addition, it

Table 4. Result of STHT, DPMI, and APMS for different models.

\begin{tabular}{|c|c|c|c|c|c|c|c|c|}
\hline \multirow{3}{*}{ DOF } & \multirow[b]{2}{*}{ Model Name } & \multicolumn{2}{|c|}{ STHT } & \multicolumn{2}{|c|}{ DPMI } & \multicolumn{2}{|c|}{ APMS } & \multirow{2}{*}{$\begin{array}{c}\text { Goodness of } \\
\text { fit average } \\
(\%)\end{array}$} \\
\hline & & $\begin{array}{c}\text { Peak fre- } \\
\text { quency (Hz) }\end{array}$ & $\begin{array}{c}\text { Goodness of } \\
\text { fit } \\
(\%) \\
\end{array}$ & $\begin{array}{c}\text { Peak fre- } \\
\text { quency (Hz) }\end{array}$ & $\begin{array}{c}\text { Goodness of } \\
\text { fit } \\
(\%) \\
\end{array}$ & $\begin{array}{c}\text { Peak fre- } \\
\text { quency (Hz) }\end{array}$ & $\begin{array}{c}\text { Goodness of } \\
\text { fit } \\
(\%) \\
\end{array}$ & \\
\hline & Target values & 5.1 & & 4.8 & & 4.4 & & \\
\hline \multirow{2}{*}{4} & Wan model & 4 & 90.9 & 7.2 & 80.1 & 3.7 & 86.8 & 85.8 \\
\hline & Optimized Wan model & 4.05 & 92 & 6.9 & 82.1 & 3.8 & 87.1 & 87 \\
\hline \multirow{2}{*}{4} & Boileau model & 4.7 & 76.8 & 5.5 & 80.1 & 4.6 & 86.7 & 81.2 \\
\hline & Optimized Boileau & 4.95 & 80.6 & 5.9 & 84 & 4.55 & 87 & 83.86 \\
\hline \multirow{2}{*}{7} & Patil model & 2.5 & 22 & 2.6 & 1 & 2.5 & $\mathbf{0}$ & 7.67 \\
\hline & Optimized Patil model & 2.1 & 35 & 2.3 & 13 & 2.1 & 34 & 27.7 \\
\hline
\end{tabular}

has the highest average of goodness of fit (87\%). So, this model is recommended for the study of biodynamic responses of seated human subjects exposed to vertical whole body vibration. The biomechanical parameters of the Wan and Schimmels model that match the experimental data was changed for the head as $c_{1}=310 \mathrm{~N} . \mathrm{s} / \mathrm{m}$ and $k_{1}=166990 \mathrm{~N} / \mathrm{m}$, respectively; and for upper torso stiffness as $k_{3}=144000 \mathrm{~N} / \mathrm{m}$. From the model, the main body resonant frequencies computed on the basis of both biodynamic response functions are found to be within close bounds to that expected for the human body.

This research provides a comprehensive understanding of the aforementioned biodynamic responses. Future research may be extended to the following:

1) Therefore, further research can be conducted on the other lumped-parameter models from literature. This work will be done after applying an optimization processes to determine the much closer one that match experimental data to STH transmissibility, DPM impedance, and AP mass values.

2) Quarter, semi, and full car suspension system including seat-human car suspension system should be analyzed and validated to find the actual frequency re- sponses of driver parts.

\section{References}

[1] G. Salvendy, "Handbook of Human Factors and Ergonomics," 2nd Edition, John Wiley \& Sons, Inc., New York, 2005.

[2] ISO 2631-1, "Mechanical Variation and Shock - Evaluation of Human Exposure to Wholebody Vibration. Part I: General Requirements," Technology Report, International Organization for Standardization, 1997.

[3] X. Wu, S. Rakheja and P.-E. Boileau, "Analyses of Relationships between Biodynamic Response Functions," Journal of Sound and Vibration, Vol. 226, No. 3, 1999, pp. 595-606.

[4] R. R. Coermann, "The Mechanical Impedance of the Human Body in Sitting and Standing Position at Low Frequencies," Human Factors, October 1962, pp. 227 253.

[5] C. W. Suggs, C. F. Abrams and L. F. Stikeleather, "Application of a Damped Spring-Mass Human Vibration Simulator in Vibration Testing of Vehicle Seats," Ergonomics, Vol. 12, No. 1, 1969, pp. 79-90.

[6] V. K. Tewari and N. Prasad, "Three-DOF Modelling of 
Tractor Seat-Operator System," Journal of Terramechanics, Vol. 36, No. 4, 1999, pp. 207-219.

[7] P. E. Boileau and S. Rakheja, "Whole-Body Vertical Biodynamic Response Characteristics of the Seated Vehicle Driver: Measurement and Model Development," International Journal of Industrial Ergonomics, Vol. 22, No. 6, 1998, pp. 449-472.

[8] Z. Zong and K. Y. Lam, "Biodynamic Response of Shipboard Sitting Subject to Ship Shock Motion," Journal of Biomechanics, Vol. 35, No. 1, 2002, pp. 35-43.

[9] X. X. Liu, J. Shi and G. H. Li, "Biodynamic Response and Injury Estimation of Ship Personnel to Ship Shock Motion Induced by Underwater Explosion," Proceeding of 69 th Shock and Vibration Symposium, Vol. 18, St. Paul, 1998, pp. 1-18.

[10] R. Muksian and C. D. Nash, "A Model for the Response of Seated Humans to Sinusoidal Displacements of the Seat," Journal of Biomechanics, Vol. 7, No. 3, 1974, pp. 209-215.

[11] M. K. Patil, M. S. Palanichamy and N. G. Dhanjoo, "Minimization of the Verticale Vibrations Sustained by a Tractor Operator, By Provision of a Standard-Type Tractor Seat Suspension," ANNALS of Biomedical Engineering, Vol. 6, 1978, pp. 138-153.

[12] C. C. Liang and C. F. Chiang, "A Study on Biodynamic Models of Seated Human Subjects Exposed to Vertical Vibration," International Journal of Industrial Ergonomics, Vol. 36, No. 10, 2006, pp. 869-890.
[13] A. E. Baumal, J. J. McPhee and P. H. Calamai, “Application of Genetic Algorithms to the Design Optimization of an Active Vehicle Suspension System," Computer Methods in Applied Mechanics and Engineering, Vol. 163, No. 1, 1998, pp. 87-94.

[14] P.-E. Boileau, "A Study of Secondary Suspensions and Human Drivers Response to Whole-Body Vehicular Vibration and Shock," Ph.D. Thesis, Concordia University, Montreal, Quebec, 1995.

[15] W. Wang, "A Study of Force-Motion and Vibration Transmission Properties of Seated Body under Vertical Vibration and Effects of Sitting Posture," Ph.D. Thesis Concordia University, Montreal, Quebec, 2006.

[16] Y. Huang, "Mechanism of Nonlinear Biodynamic Response of the Human Body Exposed to Whole-Body Vibration," Ph.D. Thesis, University of Southampton, 2008.

[17] C. C. Liang and C. F. Chiang, "Modeling of a Seated Human Body Exposed to Vertical Vibrations in Various Automotive Postures," Industrial Health, Vol. 46, No. 2, 2008, pp. 125-137.

[18] X. Wu, "A Study of Driver-Seat Interaction and Enhancement of Vehicular Ride Vibration Environment," PhD. Thesis Concordia University, Montreal, Quebec, 1998.

[19] Y. Wan and J. M. Schimmels, "A Simple Model that Captures the Essential Dynamics of a Seated Human Exposed to Whole Body Vibration," Advances in Bioengineering, ASME, Vol. 31, 1995, pp. 333-334. 